\title{
ON A BANACH SPACE OF LAPLACE-STIELTJES INTEGRALS
}

\begin{abstract}
M. M. Sheremeta, M. S. Dobushovskyy, A. O. Kuryliak. On a Banach space of Laplace-Stieltjes integrals, Mat. Stud. 48 (2017), 143-149.

Let $\Omega$ be class of positive unbounded functions $\Phi$ on $(-\infty,+\infty)$ such that the derivative $\Phi^{\prime}$ is positive, continuously differentiable and increasing to $+\infty$ on $(-\infty,+\infty), \varphi$ be the inverse function to $\Phi^{\prime}$, and $\Psi(x)=x-\frac{\Phi(x)}{\Phi^{\prime}(x)}$ be the function associated with $\Phi$ in the sense of Newton. Let $F$ be nonnegative nondecreasing unbounded continuous on the right function on $[0,+\infty)$ and $f$ be a real-value function on $[0,+\infty)$. By $L S_{\Phi}(F)$ we denote the class of integrals $I(\sigma)=$ $\int_{0}^{\infty} f(x) e^{x \sigma} d F(x)$, convergent for all $\sigma \in \mathbb{R}$ such that $|f(x)| \exp \{x \Psi(\varphi(x))\} \rightarrow 0$ as $x \rightarrow+\infty$. Put $\|I\|_{\Phi}:=\sup \{|f(x)| \exp \{x \Psi(\varphi(x))\}: x \geq 0\}$.

It is proved that if $\ln F(x)=o(x)$ as $x \rightarrow+\infty$ then $\left(L S_{\Phi}(F),\|\cdot\|_{\Phi}\right)$ is a Banach space and it is studied its properties.
\end{abstract}

1. Introduction. Let $V$ be the class of functions $F$ on $[0,+\infty)$ nonnegative nondecreasing unbounded continuous on the right. We assume that a real-value function $f$ on $[0,+\infty)$ is such that the Lebesgue-Stieltjes integral $\int_{1}^{A} f(x) e^{x \sigma} d F(x)$ exists for every $\sigma \in \mathbb{R}$ and $A \in(0,+\infty)$, and the integral

$$
I(\sigma)=\int_{0}^{\infty} f(x) e^{x \sigma} d F(x), \quad \sigma \in \mathbb{R}
$$

is called of Laplace-Stieltjes ([1]). Let

$$
M(\sigma)=M(\sigma, I)=\int_{1}^{\infty}|f(x)| e^{x \sigma} d F(x), \quad \sigma \in \mathbb{R},
$$

and

$$
\mu(\sigma)=\mu(\sigma, I)=\sup \left\{|f(x)| e^{x \sigma}: x>0\right\}, \quad \sigma \in \mathbb{R},
$$

be the maximum of the integrand. It is clear, that if $f(x) \geq 0$ for all $x \geq 0$ then $M(\sigma, I)=$ $=I(\sigma)$, and asymptotic properties of integrals of such kind are studied in the monograph [1].

By $\sigma_{M}$ we denote the abscissa of the convergence of integral (2), i. e. integral (2) converges for $\sigma<\sigma_{M}$ and diverges for $\sigma>\sigma_{M}$ (if integral (2) converges for all $\sigma \in \mathbb{R}$ then we put $\left.\sigma_{M}=+\infty\right)$. By analogy let $\sigma_{\mu}$ be the abscissa of the maximum of the integrand, i. e.

2010 Mathematics Subject Classification:26A42, 30B50.

Keywords: Laplace-Stieltjes integral; Dirichlet series; Banach space.

doi:10.15330/ms.48.2.143-149

(C) M. M. Sheremeta, M. S. Dobushovskyy, A. O. Kuryliak, 2017 
$\mu(\sigma)<+\infty$ for all $\sigma<\sigma_{\mu}$ and $\mu(\sigma)=+\infty$ for all $\sigma>\sigma_{\mu}$ (if $\mu(\sigma)<+\infty$ for all $\sigma \in \mathbb{R}$ then we put $\left.\sigma_{\mu}=+\infty\right)$.

It is easy to show $([1$, p. 8$])$ that

$$
\sigma_{\mu}=\alpha:=\varliminf_{x \rightarrow+\infty} \frac{1}{x} \ln \frac{1}{|f(x)|}
$$

and if $\ln F(x)=o(x)$ as $x \rightarrow+\infty$, then ([1, p. 13]) $\sigma_{M} \geq \sigma_{\mu}$. Hence it follows that if $\ln F(x)=o(x)$ and $\frac{1}{x} \ln \frac{1}{|f(x)|} \rightarrow+\infty$ as $x \rightarrow+\infty$ then $\sigma_{M}=+\infty$. The inequality $\sigma_{M} \leq \sigma_{\mu}$ is perhaps incorrect $([1, \mathrm{p} .21])$.

Here we consider the case $\sigma_{M}=\sigma_{\mu}=+\infty$ and by $\Omega$ denote the class of positive functions $\Phi$ unbounded on $(-\infty,+\infty)$ such that the derivative $\Phi^{\prime}$ is positive continuously differentiable and increasing to $+\infty$ on $(-\infty,+\infty)$. It is clear that the function $\Phi \in \Omega$ is convex on $(-\infty,+\infty), \Phi(x) \rightarrow c \geq 0(x \rightarrow-\infty)$ and $\Phi^{\prime}(x) \rightarrow 0(x \rightarrow-\infty)$. From now on, we denote by $\varphi$ the inverse function to $\Phi^{\prime}$, and let $\Psi(x)=x-\frac{\Phi(x)}{\Phi^{\prime}(x)}$ be the function associated with $\Phi$ in the sense of Newton. It is clear that the function $\varphi$ is continuously differentiable and increasing to $+\infty$ on $(0,+\infty)$. The function $\Psi$ is $([1$, p. 30]) continuously differentiable and increasing to $+\infty$ on $(-\infty,+\infty)$. The following lemma is true ([1, p. 30]).

Lemma 1. Let $\Phi \in \Omega$. In order that $\ln \mu(\sigma) \leq \Phi(\sigma)$ for all $\sigma \in\left(\sigma_{0},+\infty\right)$, it is necessary and sufficient that $\ln f(x) \leq-x \Psi(\varphi(x))$ for all $x \geq x_{0}$.

2. Banach space of Laplace-Stiltijes integrals. Let $\Phi \in \Omega$ and

$$
J(\sigma)=\int_{0}^{\infty} \exp \{-x \Psi(\varphi(x))\} e^{x \sigma} d F(x) .
$$

Since

$$
\frac{1}{x} \ln \frac{1}{\exp \{-x \Psi(\varphi(x))\}}=\Psi(\varphi(x)) \rightarrow+\infty, \quad x \rightarrow+\infty
$$

integral (4) converges for all $\sigma \in(-\infty,+\infty)$. By $L S_{\Phi}(F)$ we denote the class of integrals (1) with real-valued functions $f$ such that

$$
|f(x)| \exp \{x \Psi(\varphi(x))\} \rightarrow 0, \quad x \rightarrow+\infty .
$$

On $L S_{\Phi}(F)$ we define operations

$$
\left(I_{1}+I_{2}\right)(\sigma)=\int_{0}^{\infty}\left(f_{1}(x)+f_{1}(x)\right) e^{x \sigma} d F(x), \quad(\lambda I)(\sigma)=\int_{0}^{\infty} \lambda f(x) e^{x \sigma} d F(x),
$$

where

$$
I_{p}(\sigma)=\int_{0}^{\infty} f_{p}(x) e^{x \sigma} d F(x)
$$

where $p=1,2$ and $\lambda$ is a real number, and let

$$
\|I\|_{\Phi}:=\sup \{|f(x)| \exp \{x \Psi(\varphi(x))\}: x \geq 0\} .
$$

Under these operations $L S_{\Phi}(F)$ becomes a normed linear space. 
Theorem 1. If $F \in V$ and $\ln F(x)=o(x)$ as $x \rightarrow+\infty$ then $\left(L S_{\Phi}(F)\right.$, $\left.\|\cdot\|_{\Phi}\right)$ is nonuniformly convex Banach space.

Proof. Let $\left(I_{p}\right)$ be a Cauchy sequence in $L S_{\Phi}(F)$ such that $I_{p}(\sigma)$ is defined by (6). Then $\left|f_{p}(x)\right| \exp \{x \Psi(\varphi(x))\} \rightarrow 0$ as $x \rightarrow+\infty$ for each $p$. As $\left(I_{p}\right)$ is Cauchy sequence so for a given $\varepsilon>0$ there exits $n_{0}=n_{0}(\varepsilon) \in \mathbb{N}$ such that $\left\|I_{p}-I_{q}\right\|_{\Phi}<\varepsilon$ for all $p \geq n_{0}$ and $q \geq n_{0}$, i. e.

$$
\sup \left\{\left|f_{p}(x)-f_{q}(x)\right| \exp \{x \Psi(\varphi(x))\}: x \geq 0\right\}<\varepsilon
$$

for all $p \geq n_{0}$ and $q \geq n_{0}$, and, thus,

$$
\left|f_{p}(x) \exp \{x \Psi(\varphi(x))\}-f_{q}(x) \exp \{x \Psi(\varphi(x))\}\right|<\varepsilon
$$

for all $x \geq 0, p \geq n_{0}$ and $q \geq n_{0}$. This shows that $\left(f_{p}(x) \exp \{x \Psi(\varphi(x))\}\right)$ is Cauchy sequence in $\mathbb{R}$, so converges to $f_{0}(x) \exp \{x \Psi(\varphi(x))\}$ (say) as $p \rightarrow \infty$. Since

$$
\begin{gathered}
\left|f_{0}(x)\right| \exp \{x \Psi(\varphi(x))\}= \\
=\left|f_{0}(x) \exp \{x \Psi(\varphi(x))\}-f_{p}(x) \exp \{x \Psi(\varphi(x))\}+f_{p}(x) \exp \{x \Psi(\varphi(x))\}\right| \leq \\
\leq\left|f_{0}(x) \exp \{x \Psi(\varphi(x))\}-f_{p}(x) \exp \{x \Psi(\varphi(x))\}\right|+\left|f_{p}(x)\right| \exp \{x \Psi(\varphi(x))\} \rightarrow 0, \quad x \rightarrow+\infty,
\end{gathered}
$$

the integral

$$
I_{0}(\sigma)=\int_{0}^{\infty} f_{0}(x) e^{x \sigma} d F(x)
$$

belongs to $L S_{\Phi}(F)$. Further,

$$
\left\|I_{p}-I_{0}\right\|_{\Phi}=\sup \left\{\left|f_{p}(x)-f_{0}(x)\right| \exp \{x \Psi(\varphi(x))\}: x \geq 0\right\} \rightarrow 0, \quad p \rightarrow \infty,
$$

i. e. the $\left(L S_{\Phi}(F),\|I\|_{\Phi}\right)$ is complete and, therefore, a Banach space.

Now we choose numbers $0<a_{n}<b_{n}<c_{n}<+\infty$ such that

$$
\int_{0}^{a_{n}} \exp \{-x \Psi(\varphi(x))\} e^{x \sigma} d F(x) \neq 0, \quad \int_{b_{n}}^{c_{n}} \exp \{-x \Psi(\varphi(x))\} e^{x \sigma} d F(x) \neq 0
$$

and put

$$
I_{n}(\sigma)=\int_{0}^{a_{n}} \exp \{-x \Psi(\varphi(x))\} e^{x \sigma} d F(x)
$$

and

$$
J_{n}(\sigma)=\int_{0}^{a_{n}} \exp \{-x \Psi(\varphi(x))\} e^{x \sigma} d F(x)+\int_{b_{n}}^{c_{n}} \exp \{-x \Psi(\varphi(x))\} e^{x \sigma} d F(x) .
$$

Then $I_{n} \in L S_{\Phi}(F), J_{n} \in L S_{\Phi}(F),\left\|I_{n}\right\|=1,\left\|J_{n}\right\|=1,\left\|J_{n}+I_{n}\right\|=2$, but $\left\|J_{n}-I_{n}\right\|=1 \not \rightarrow 0$, i. e. the space $\left(L S_{\Phi}(F),\|\cdot\|_{\Phi}\right)$ is non-uniformly convex (see. for example, [2, p. 183]).

Remark 1. From (5) it follows that $\ln |f(x)| \leq-x \Psi(\varphi(x))$ for all $x \geq x_{0}$. Therefore, by Lemma $1 \ln \mu(\sigma, I) \leq \Phi(\sigma)$ for all $\sigma \in\left(\sigma_{0},+\infty\right)$ for every $I \in L S_{\Phi}(F)$. On the other hand, if $\ln F(x)=O(\Phi(\Psi(\varphi(x))))$ as $x \rightarrow+\infty$ and $\ln \mu(\sigma, I) \leq \Phi(\sigma)$ for all $\sigma \in\left(\sigma_{0},+\infty\right)$ then [1, p. 103] $\ln I(\sigma) \leq(1+o(1)) \Phi(\sigma)$ as $\sigma \rightarrow+\infty$, i. e. $\varlimsup_{\sigma \rightarrow+\infty} \frac{\ln I(\sigma)}{\Phi(\sigma)} \leq 1$ for each $I \in L S_{\Phi}(F)$. 
The following statement concerns for uniform convergence.

Proposition 1. Let $F \in V$ and $\ln F(x)=o(x)$ as $x \rightarrow+\infty$. If $\left(I_{m}\right) \subset L S_{\Phi}(F)$ converges to $I \in L S_{\Phi}(F)$ by $\|\cdot\|_{\Phi}$, then $I_{m}(\sigma)$ converges uniformly to $I(\sigma)$ over compact subset of $\mathbb{R}$.

Proof. If $\left\|I_{p}-I\right\|_{\Phi}<\varepsilon$ for every $\varepsilon>0$ and all $p \geq p_{0}(\varepsilon)$ then

$$
\sup \left\{\left|f_{p}(x)-f(x)\right| \exp \{x \Psi(\varphi(x))\}: x \geq 0\right\}<\varepsilon
$$

and, thus, $\left|f_{p}(x)-f(x)\right| \exp \{x \Psi(\varphi(x))\}<\varepsilon$ for every $\varepsilon>0$, all $p \geq p_{0}(\varepsilon)$ and all $x \geq 0$. Therefore, if $p \geq p_{0}(\varepsilon)$ and $\sigma \in\left[\sigma_{1}, \sigma_{2}\right]$ then in view of condition $\ln F(x)=o(x)$ as $x \rightarrow+\infty$ we have

$$
\begin{aligned}
& \left|I_{p}(\sigma)-I(\sigma)\right|<\int_{0}^{+\infty}\left|f_{p}(x)-f(x)\right| \exp \{x \Psi(\varphi(x))\} \exp \{-x \Psi(\varphi(x))\} e^{x \sigma} d F(x)< \\
& \quad<\varepsilon \int_{0}^{+\infty} \exp \{-x \Psi(\varphi(x))\} e^{x \sigma_{2}} d F(x)=\varepsilon \int_{0}^{+\infty} e^{-x\left(\Psi(\varphi(x))-\sigma_{2}\right)} d F(x) \leq \\
& \leq \varepsilon \int_{0}^{+\infty} F(x) d e^{-x\left(\Psi(\varphi(x))-\sigma_{2}\right)} \leq \varepsilon \int_{0}^{+\infty} e^{K_{1} x} d e^{-x\left(\Psi(\varphi(x))-\sigma_{2}\right)} \leq K_{2} \varepsilon, \quad K=\text { const } .
\end{aligned}
$$

Therefore, $\left(I_{p}(\sigma)\right)$ converges uniformly to $I(\sigma)$ on $\left[\sigma_{1}, \sigma_{2}\right]$.

Remark 2. The converse statement to Proposition 1 is false. Indeed, let for every $m \in \mathbb{Z}_{+}$ and $n \in \mathbb{N}$

$$
F(x)=\left\{\begin{array}{l}
0,1 \leq x<2 \\
n, 2 n \leq x<2(n+1),
\end{array} \quad f_{m}(x)=\left\{\begin{array}{l}
\alpha_{m, n}>0, x=2 n-1 \\
0, x \neq 2 n-1
\end{array}\right.\right.
$$

Then for all $m \in \mathbb{Z}_{+}$

$$
I_{m}(\sigma)=\int_{0}^{\infty} f_{m}(x) e^{x \sigma} d F(x)=\sum_{n} f_{m}(2 n) e^{2 n \sigma}=0
$$

i. e. $I_{m}(\sigma) \rightarrow I_{0}(\sigma)$ for all $\sigma \in\left[\sigma_{1}, \sigma_{2}\right]$. On the other hand,

$$
\begin{aligned}
& \left\|I_{m}-I_{0}\right\|_{\Phi}=\sup \left\{\left|f_{m}(x)-f_{0}(x)\right| \exp \{-x \Psi(\varphi(x))\}: x \geq 0\right\} \geq \\
& \geq\left|f_{m}(1)-f_{0}(1)\right| \exp \{\Psi(\varphi(1))\}=\left|\alpha_{m, 1}-\alpha_{0,1}\right| \exp \{\Psi(\varphi(1))\} \geq h_{1}>0
\end{aligned}
$$

provided $\alpha_{m, 1}-\alpha_{0,1} \geq \eta_{1}>0$ for all $m \in \mathbb{N}$.

For $\left(L S_{\Phi}(F),\|\cdot\|_{\Phi}\right)$ by $L S_{\Phi}(F)^{*}$ we denote the dual space, i. e. $L S_{\Phi}(F)^{*}$ is the family of all continuous linear functionals on $\left(L S_{\Phi}(F),\|\cdot\|_{\Phi}\right)$.

Let $\Lambda(I)=\int_{1}^{\infty} f(x) g(x) d F(x)$, where $g$ is a real-valued function on $(1,+\infty)$ such that $\int_{1}^{\infty}|f(x) g(x)| d F(x)<+\infty$. Then $\Lambda$ is a linear functional and the following proposition is true. 
Proposition 2. In order that $\Lambda \in L S_{\Phi}(F)^{*}$ it is sufficient that

$$
\int_{1}^{\infty}|g(x)| \exp \{-x \Psi(\varphi(x))\} d F(x)<+\infty .
$$

Proof. By definition we have

$$
\begin{gathered}
\|\Lambda(I)\|_{\Phi}=\sup \left\{|\Lambda(I)|:\|I\|_{\Phi} \leq 1\right\}= \\
=\sup \left\{\int_{0}^{\infty}|f(x)| e^{x \Psi(\varphi(x))}|g(x)| e^{-x \Psi(\varphi(x))} d F(x): \sup _{x>0} \mid f(x) e^{x \Psi(\varphi(x))} \leq 1\right\} \leq \\
\leq \int_{1}^{\infty}|g(x)| \exp \{-x \Psi(\varphi(x))\} d F(x)<+\infty,
\end{gathered}
$$

i. e. $\Lambda \in L S_{\Phi}(F)^{*}$. Proposition 2 is proved.

Conjecture. Every bounded linear functional $\Lambda$ defined for $I \in\left(L S_{\Phi}(F),\|\cdot\|_{\Phi}\right)$ is of the form

$$
\Lambda(I)=\int_{1}^{\infty} f(x) g(x) d F(x), \quad I(\sigma)=\int_{0}^{\infty} f(x) e^{x \sigma} d F(x),
$$

where $g(x)$ satisfies $(7)$.

Below we prove this conjecture for entire Dirichlet series.

3. Banach space of Dirichlet series. Let $0<\lambda_{n} \uparrow \infty$ and $n(x)=\sum_{\lambda_{n} \leq x} 1$ be a counting function of the sequence $\lambda=\left(\lambda_{n}\right)$. If we choose $f(x)=a_{n}$ for $x=\lambda_{n}$ and $f(x)=0$ for $x \neq \lambda_{n}$ and $F(x)=n(x)$ then from (1) we obtain a Dirichlet series

$$
D(\sigma)=\sum_{n=1}^{\infty} a_{n} e^{\lambda_{n} \sigma}
$$

By $S(\lambda, \Phi)$ we denote a class of entire Dirichlet series (8) with real-valued coefficients $a_{n}$ such that

$$
\left|a_{n}\right| \exp \left\{\lambda_{n} \Psi\left(\varphi\left(\lambda_{n}\right)\right)\right\} \rightarrow 0, \quad n \rightarrow+\infty
$$

and we put

$$
\|D\|_{\Phi}:=\sup \left\{\left|a_{n}\right| \exp \left\{\lambda_{n} \Psi\left(\varphi\left(\lambda_{n}\right)\right)\right\}: n \geq 1\right\} .
$$

Then from Theorem 1 we obtain the following statement.

Corollary 1. If $\ln n(x)=o(x)$ as $x \rightarrow+\infty$ then $\left(S(\lambda, \Phi),\|\cdot\|_{\Phi}\right)$ is non-uniformly convex Banach space.

The following theorem completes Proposition 1.

Theorem 2. If $\ln n(x)=o(x)$ as $x \rightarrow+\infty$ then in order that $\left(D_{m}\right) \subset S(\lambda, \Phi)$ converges to $D \in S(\lambda, \Phi)$ by $\|\cdot\|_{\Phi}$ it is necessary and sufficient that $D_{m}(\sigma)$ converges uniformly to $D(\sigma)$ over each compact subset of $\mathbb{R}$. 
Proof. The necessity follows from Proposition 1. Conversely, let $\left|D_{m}(\sigma)-D(\sigma)\right|<\varepsilon$ for all $\sigma \in B=\left[\sigma_{1}, \sigma_{1}\right]$ and all $m \geq m_{0}=m_{0}(\varepsilon, B)$, where $D_{m}(\sigma)=\sum_{n=1}^{\infty} a_{m, n} e^{\lambda_{n} \sigma}$. Then $\left|a_{m, n}-a_{n}\right| e^{\lambda_{n} \sigma_{1}}<\varepsilon$ for all $n \geq 1$ and all $m \geq m_{0}=m_{0}\left(\varepsilon, \sigma_{1}\right)$, whence

$$
\left|a_{m, n}-a_{n}\right| \exp \left\{\lambda_{n} \Psi\left(\varphi\left(\lambda_{n}\right)\right)\right\} \leq \varepsilon \exp \left\{-\left(\sigma_{1}-\lambda_{n} \Psi\left(\varphi\left(\lambda_{n}\right)\right)\right)\right\} .
$$

Choosing $\sigma_{1}=\lambda_{n} \Psi\left(\varphi\left(\lambda_{n}\right)\right)$ hence we obtain that $\left|a_{m, n}-a_{n}\right| \exp \left\{\lambda_{n} \Psi\left(\varphi\left(\lambda_{n}\right)\right)\right\} \leq \varepsilon$ for every $\varepsilon>0$, all $n \geq 1$ and all $m \geq m_{0}=m_{0}(\varepsilon, n)$, i. e. $\left\|D_{m}-D\right\|_{\Phi} \rightarrow 0$ as $m \rightarrow \infty$.

For $\left(S(\lambda, \Phi),\|\cdot\|_{\Phi}\right)$ by $S(\lambda, \Phi)^{*}$ we denote the dual space and put $\Lambda(D)=\sum_{n=1}^{\infty} a_{n} g_{n}$, where $\left(g_{n}\right)$ is a real-valued sequence such that $\sum_{n=1}^{\infty}\left|g_{n}\right| \exp \left\{-\lambda_{n} \Psi\left(\varphi\left(\lambda_{n}\right)\right)\right\}<+\infty$. Then $\Lambda$ is a linear functional and the following proposition is true.

Theorem 3. Every bounded linear functional $\Lambda$ defined for $\left(S(\lambda, \Phi),\|\cdot\|_{\Phi}\right)$ is of the form

$$
\Lambda(D)=\sum_{n=1}^{\infty} a_{n} g_{n}, \quad D(\sigma)=\sum_{n=1}^{\infty} a_{n} e^{\lambda_{n} \sigma}
$$

where $\left(g_{n}\right)$ is a real-valued sequence such that $\sum_{n=1}^{\infty}\left|g_{n} \exp \left\{-\lambda_{n} \Psi\left(\varphi\left(\lambda_{n}\right)\right)\right\}\right|<+\infty$.

Proof. We use a method from [3]. As in the proof of Proposition 2 now we have

$$
\begin{gathered}
\sum_{n=1}^{\infty}\left|a_{n} g_{n}\right| \leq \sup \left\{\left|a_{n}\right| \exp \left\{\lambda_{n} \Psi\left(\varphi\left(\lambda_{n}\right)\right)\right\} \sum_{n=1}^{\infty}\left|g_{n}\right| \exp \left\{-\lambda_{n} \Psi\left(\varphi\left(\lambda_{n}\right)\right)\right\}=\right. \\
=\|D\| \sum_{n=1}^{\infty}\left|g_{n}\right| \exp \left\{-\lambda_{n} \Psi\left(\varphi\left(\lambda_{n}\right)\right)\right\}<+\infty
\end{gathered}
$$

i. e. $\Lambda$ is well defined functional on $\left(S(\lambda, \Phi),\|\cdot\|_{\Phi}\right)$. Moreover,

$$
|\Lambda(D)| \leq\|D\| \sum_{n=1}^{\infty}\left|g_{n}\right| \exp \left\{-\lambda_{n} \Psi\left(\varphi\left(\lambda_{n}\right)\right)\right\}
$$

whence

$$
\|\Lambda\| \leq \sum_{n=1}^{\infty}\left|g_{n}\right| \exp \left\{-\lambda_{n} \Psi\left(\varphi\left(\lambda_{n}\right)\right)\right\} .
$$

Conversely, first we remark that if $\left.D \in S(\lambda, \Phi),\|\cdot\|_{\Phi}\right)$ and $D_{m}(\sigma)=\sum_{n=1}^{m} a_{n} e^{\lambda_{n} \sigma}$ then

$$
\left\|D_{m}-D\right\|_{\Phi}=\sup _{n>m}\left\{\left|a_{n}\right| \exp \left\{\lambda_{n} \Psi\left(\varphi\left(\lambda_{n}\right)\right)\right\}\right\} \rightarrow 0
$$

as $m \rightarrow \infty$ and by Theorem $2 D_{m}(\sigma)$ converges uniformly to $D(\sigma)$ over each compact subset of $\mathbb{R}$. Therefore, if $\Lambda \in S(\lambda, \Phi)^{*}$ and be defined as $\Lambda\left(e^{\sigma \lambda_{n}}\right)=g_{n}$ for each $n$ then

$$
\Lambda(D)=\Lambda\left(\lim _{m \rightarrow \infty} \sum_{n=1}^{m} a_{n} e^{\lambda_{n} \sigma}\right)=\lim _{m \rightarrow \infty} \sum_{n=1}^{m} a_{n} L\left(e^{\lambda_{n} \sigma}\right)=\sum_{n=1}^{\infty}\left|a_{n} g_{n}\right| .
$$

Now we show that $\sum_{n=1}^{\infty}\left|a_{n} g_{n}\right| \leq\|\Lambda\| \mid$ so that $\sum_{n=1}^{\infty}\left|a_{n} g_{n}\right|<+\infty$. For it take any $p \geq 1$ and let $p \in \mathbb{N}$ and

$$
a_{n}= \begin{cases}\exp \left\{-\lambda_{n} \Psi\left(\varphi\left(\lambda_{n}\right)\right)\right\} \operatorname{sgn}\left(g_{n}\right), & 1 \leq n \leq p \\ 0, & n>p\end{cases}
$$


If we define $D(\sigma)=\sum_{n=1}^{\infty} a_{n} \exp \left\{\sigma \lambda_{n}\right\}$ then obviously $D \in S(\lambda, \Phi)$ and $\|D\|_{\Phi}=1$. Hence

$$
|\Lambda(D)|=\left|\sum_{n=1}^{p} \exp \left\{-\lambda_{n} \Psi\left(\varphi\left(\lambda_{n}\right)\right)\right\} \operatorname{sgn}\left(g_{n}\right) \Lambda\left(e^{\sigma \lambda_{n}}\right)\right|=\sum_{n=1}^{p} \exp \left\{-\lambda_{n} \Psi\left(\varphi\left(\lambda_{n}\right)\right)\right\}\left|g_{n}\right|
$$

and $|\Lambda(D)| \leq\|\Lambda\|\|D\|=\|\Lambda\|$, so that $\sum_{n=1}^{p} \exp \left\{-\lambda_{n} \Psi\left(\varphi\left(\lambda_{n}\right)\right)\right\}\left|g_{n}\right| \leq\|\Lambda\|$ and

$$
\sum_{n=1}^{\infty} \exp \left\{-\lambda_{n} \Psi\left(\varphi\left(\lambda_{n}\right)\right)\right\}\left|g_{n}\right| \leq \sup _{p} \sum_{n=1}^{p} \exp \left\{-\lambda_{n} \Psi\left(\varphi\left(\lambda_{n}\right)\right)\right\}\left|g_{n}\right| \leq \sup _{p}\|\Lambda\|=\|\Lambda\| \text {. }
$$

Inequalities (11) and (12) together show that $\sum_{n=1}^{\infty} \exp \left\{-\lambda_{n} \Psi\left(\varphi\left(\lambda_{n}\right)\right)\right\}\left|g_{n}\right|=\|\Lambda\|$ and this completes the proof of the theorem.

\section{REFERENCES}

1. Sheremeta M.M., Asymptotical behaviour of Laplace-Stiltjes integrals, Lviv: VNTL Publishers, 2010, $211 \mathrm{p}$.

2. Trenogin V.A., Functional analysis. M.: Nauka, 1980, 495 p. (in Russian)

3. Juneja O.P., Srivastava B.L. On a Banach space of a class of Diriclet series // Indian J. pure appl. Math. - 1981. - V.12, №4. - P. 521-529.

Ivan Franko National University of Lviv

m_m_sheremeta@gmail.com

mōobush19@gmail.com

andriykuryliak@gmail.com 